\title{
Voronoi Diagram Based Optimization of Dynamic Reactive Power Sources
}

\author{
Weihong Huang, Kai Sun, Junjian Qi \\ University of Tennessee \\ Knoxville, TN, USA \\ whuang12@utk.edu, kaisun@utk.edu, \\ junjian.qi.2012@ieee.org
}

\author{
Yan $\mathrm{Xu}$ \\ Oak Ridge National Laboratory \\ Oak Ridge, TN, USA \\ xuy3@ornl.gov
}

\begin{abstract}
Dynamic var sources can effectively mitigate faultinduced delayed voltage recovery (FIDVR) issues or even voltage collapse. This paper proposes a new approach to optimization of the sizes of dynamic var sources at candidate locations by a Voronoi diagram based algorithm. It first disperses sample points of potential solutions in a searching space, evaluates a cost function at each point by barycentric interpolation for the subspaces around the point, and then constructs a Voronoi diagram about cost function values over the entire space. Accordingly, the final optimal solution can be obtained. Case studies on the WSCC 9-bus system and NPCC 140-bus system have validated that the new approach can quickly identify the boundary of feasible solutions in searching space and converge to the global optimal solution.
\end{abstract}

Index Terms--dynamic var support; FIDVR; nonlinear optimization; Voronoi Diagram

\section{INTRODUCTION}

There are increasing interests in optimization of dynamic var sources in a power system due to growing concerns with dynamic voltage security issues, especially fault-induced delayed voltage recovery (FIDVR) issues and even fast voltage collapse at load buses. Some papers have studied the optimal allocation of dynamic var sources [1]-[5]. In [1] and [2], the dynamic var allocation problem is formulated as a mixed integer nonlinear programing problem. The sizes of dynamic var are optimized by interfacing the solution of that nonlinear programming problem with power system timedomain simulation software to ensure the post-contingency voltage responses meet reliability criteria. The approach in [3] solves the same problem by interfacing heuristic linear programming with power system simulation, which takes a small number of iterations to converge to an optimal solution from an initial guess. The approach of [4] employs the meanvariance mapping optimization in combination with an integrated mix-integer search strategy and two intervention schemes. The method in [5] applies multi-objective evolutionary algorithm to optimal sizing of dynamic var sources. Both [4] and [5] addresses preselected sets of shortterm voltage stability issues.

This manuscript has been authored by UT-Battelle, LLC, under Contract No. DE-AC05-00OR22725 with the U.S. Department of Energy. The United States Government retains and the publisher, by accepting the article for publication, acknowledges that the United States Government retains a nonexclusive, paid-up, irrevocable, world-wide license to publish or reproduce the published form of this manuscript, or allow others to do so, for United States Government purposes.
The complexity of the optimal allocation of dynamic var sources is mainly because the problem itself is a nonlinear optimization problem and checking its constraints requires knowing post-fault power system trajectories. Thus, both a nonlinear optimization solver and a power system differential algebraic equation (DAE) solver are required and they should be systematically integrated or interfaced to solve the problem after a number of iterations. Computation burdens exist with both solvers. Also to ensure the iterations to converge to the global optimum, the entire feasible solutions may need to exhaustively searched, which is non-convex for most cases. An idea to overcome that problem is to estimate the boundary of a region of feasible solutions including the global optimum and then depict the region's contour map that is colored by the value of a cost function for different solution. Based on such a contour map, the global optimum may easily be founded by a small number of iterations.

Voronoi diagram [6] is one of the most useful data structures in computational geometry and an effective technique for generating contour map. It has been widely applied to diverse fields of science and engineering. In [7], a Voronoi diagram based blending method of local response surfaces was proposed to accomplish global fidelity in function approximation for engineering designing optimization. The method compromised the tradeoffs between local and global properties in function approximation through adaptive accumulation of sample points based on Voronoi diagram geometric information on their distribution. Additionally, the approximation was gradually refined in ever smaller Voronoi regions with quadratic polynomial expression.

In this paper, a Voronoi diagram based scheme is proposed for optimization of the sizes of dynamic var sources at predetermined locations. The Voronoi diagram technique is applied by the scheme to generate a contour map about the optimality and feasibility of any point in a searching space for solutions. The proposed scheme first simulates for a certain number of potential solutions, i.e. sample points in the searching space for dynamic var sizes. Second, an approximate value for the objective function is calculated 
based on the simulation result for each point. Third, an optimal solution is sought by piling barycentric interpolation [8] to get a local approximation function for global response surface instead of direct analysis. Finally, the Voronoi diagram is modeled to distribute sample points until getting global fidelity. The proposed approach is tested on the WSCC 9-bus system and the NPCC (Northeastern Power Coordinating Council) 140-bus power system model.

\section{VORONOI DIAGRAM BASED BLENDING OF LOCAL FUNCTION EXPRESSION}

The approximation of a function for design optimization is based on local information at a given set of sample points. The Voronoi diagram and barycentric interpolation method are used for estimation of local information and described as follows.

\section{A. Voronoi Diagram}

Denote the Euclidean distance between two points $p$ and $q$ by $\operatorname{dis}(p, q)=\sqrt{\left(p_{x}-q_{x}\right)^{2}+\left(p_{y}-q_{y}\right)^{2}}$. Let $P=$ $\left\{p_{1}, p_{2}, \cdots p_{10}\right\}$ be a set of $n$ distinct points in a plane as shown in Figure 1(a). Perpendicular bisectors are drawn for each pair of points shown as triangular edge in Figure 1(b). This results in a set of polygons which will actually be polyhedra for a higher dimensional space. These polygons as Voronoi regions of $P_{i}$ are denoted as $\operatorname{Vor}\left(P_{i}\right)$. One for each site in $P$, with the property that a point $q$ lies in the cell corresponding to a site $p_{i}$ if and only if $\operatorname{dis}\left(q, p_{i}\right)<$ $\operatorname{dis}\left(q, p_{j}\right)$ for each $p_{j} \in P$ with $j \neq i$. All of the intersection points $q_{1}, q_{2}, \ldots, q_{9}, \ldots$ are named Voronoi points.

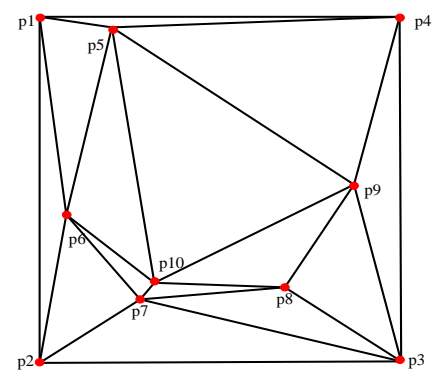

(a)

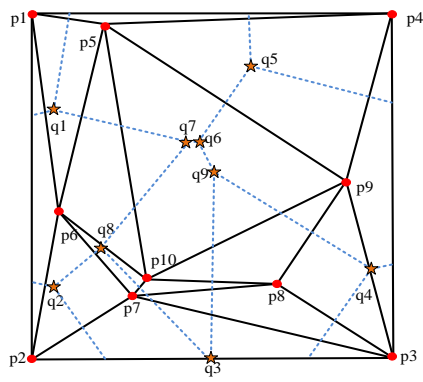

(b)
Figure 1. Voronoi diagram.

\section{B. Barycentric Interpolation}

Barycentric interpolation is a variant of Lagrange polynomial interpolation which is also known as areal coordinates. Barycentric interpolation is useful especially in engineering applications involving triangular subdomains. Taking the triangle composited by $p_{5}, p_{9}$, and $p_{10}$ as an example, its three vertices have three objective values as shown in Figure 2. Each point located inside this triangle can be written as a unique convex combination of the three vertices. In other words, for each point there is a unique sequence of three numbers. If we want to estimate the objective function value of $q_{9}$, there is a unique sequence of three numbers, $\alpha_{1}, \alpha_{2}$, and $\alpha_{3} \geq 0$ such that $\alpha_{1}+\alpha_{2}+\alpha_{3}=$ 1 and $q_{9}=\alpha_{1} p_{5}+\alpha_{2} p_{10}+\alpha_{3} p_{9} . \alpha_{1}, \alpha_{2}, \alpha_{3}$ indicate the barycentric or area coordinates of the point $q_{9}$ with respect to the triangle. Every point inside the triangle of $p_{5}, p_{9}, p_{10}$ is uniquely defined by any two of the barycentric coordinates. There are $\alpha_{1}=\frac{A 1}{A 1+A 2+A 3}, \alpha_{2}=\frac{A 2}{A 1+A 2+A 3}$ and $\alpha_{3}=\frac{A 3}{A 1+A 2+A 3}$

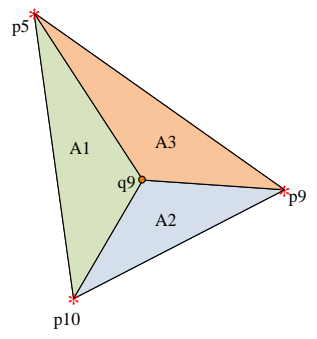

Figure 2. Barycentric interpolation.

\section{PROPOSED APPROACH}

When a new sample data is added to the established approximation function, after its small neighboring parts are arranged under the characteristic of Voronoi diagram, the approximation function is reformed with less cost. This section describes how this approximation scheme is combined into an adaptive global optimization computation with optimization of dynamic var sources.

\section{A. Placement and Optimization for Dynamic Var Sources}

Since the placement of dynamic var sources is not a focus of this paper, their locations are assumed to be determined by a voltage sensitivity index (VSI), where var injection will cause more sensitive improvements on the overall post-fault voltage level [3]. Other methods or engineering criteria on the placement of dynamic var sources may also apply.

This paper considers typical voltage criteria according to the WECC/NERC planning standards [9] to formulate the following objective function for optimization. Assume the system has $N$ buses. Define the percentage voltage deviation of a bus $j$ at time $t$ :

$$
R_{j}=\left|\frac{V_{j}(t)-V_{j}^{\text {init }}}{V_{j}^{\text {init }}}\right| \times 100 \%, \forall j \in 1 \sim N
$$

where $V_{j}^{\text {init }}$ is the pre-fault initial voltage magnitude. Thus, the optimal allocation problem for dynamic var sources can be formulated as follows.

Minimize

Subject to

$$
f=\sum_{i \in I_{Q}} c_{i} Q_{i}+c_{p} Z
$$

$$
\begin{aligned}
& Q_{i}^{L} \leq Q_{i} \leq Q_{i}^{U}, \forall i \in I_{Q} \\
& R_{j} \leq 25 \%, t_{c l} \leq t<t_{s}, \forall j \in I_{L} \\
& R_{j} \leq 30 \%, t_{c l} \leq t<t_{s}, \forall j \in I_{G} \\
& R_{j} \leq 5 \%, t \geq t_{s}, \forall j \in 1 \sim N \\
& \text { Duration }_{R_{j} \geq 20 \%}<20 \text { cycles, } t_{c l} \leq t<t_{s}, \forall j \in I_{L} \\
& \dot{x}=f(x, y, Q)
\end{aligned}
$$

where $t_{c l}$ is the fault clearing time, $t_{s}$ is the post transit time, $Q_{i}$ is the dynamic var injected at candidate bus $i, c_{i}$ is the cost coefficient associated with $Q_{i}$, e.g. the cost per Mvar, $Z$ is binary (=0 when not violating the standard; $=1$ otherwise), $c_{p}$ is the penalty cost, $Q_{i}{ }^{L}$ and $Q_{i}{ }^{U}$ are the lower and upper limits of $Q_{i}, I_{Q}$ is the set of candidate buses, $I_{L}$ and $I_{G}$ are respectively the sets of load and generator buses, equation (8) 
represents the DAEs on power system dynamics, $x$ and $y$ are vectors of differential variables and algebraic variables, and $Q$ is the vector of $Q_{i}$.

\section{B. Proposed Algorithm for Optimization}

The overall algorithm for tackling the area is configured into following steps:

Step 1. Perform certain number of initial random sample points and evaluate cost functions at those points.

Step 2. Establish or update the Voronoi diagram and estimate the objective function by barycentric interpolation for a neighborhood of each sample point.

Step 3. Find a tentative solution by solving an approximated objective function.

Step 4. If a solution has enough fidelity or if the cost exceeds the limit, terminate the overall procedure.

Step 5. Determine new sample points, and do power system simulation.

Step 6. Go to Step 2

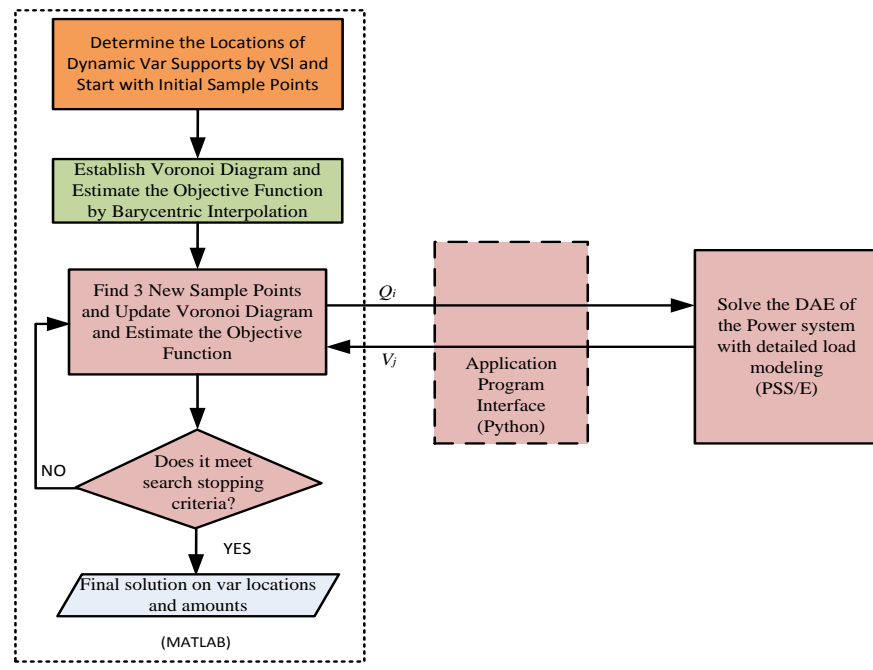

Figure 3. Flow chart for implementing the proposed approach.

Figure 3 illustrates the implementation of the approach, which interfaces the Voronoi diagram (performed in MATLAB) with power system dynamics simulator PSS/E via Python. PSS/E solves the DAEs in (8) to simulate the postfault voltage responses for any given var injection strategy. The results are fed back to the Voronoi diagram to check voltage criteria and update the diagram. Induction motor loads and SVCs need to be modeled in PSS/E simulations.

\section{Adding New Sample Points}

An $n$-dimensional space requires at least $n+1$ points to establish a Voronoi diagram. To make the diagram have additional geometrical characteristics, more points should be utilized [10]. In this paper, $2(n+1)$ initial sample points are randomly selected in the searching region together with its $2^{n}$ geometric vertices (i.e. the limits in all dimensions) to build the first Voronoi diagram approximating the entire region. Then, to improve global approximation fidelity, the following three new sample points should recursively be added at each execution of Step 5. Since the proposed approach gradually adds new points to refine the Voronoi diagram, especially for the area surrounding the global optimum, the final result is not sensitive to the selection of the initial $2(n+1)$ sample points, which are confirmed by our tests running the approach with different groups of initial sample points.

\section{1) New point 1 for optimization}

The triangle where the tentative optimal solution is obtained is considered as the most effective area. A new candidate sample point shown as $p_{n}$ in Figure 4 is chosen based on the minimum objective function from the barycentric interpolation. Considering interpolation value will be changed by addition of other points, a new sample point is arranged as $p_{n}{ }^{*}$ which is randomly generated within the tentative solution triangle $\Delta q_{8} p_{10} q_{3}$ (polygonal pyramid in the higher dimensional) where $p_{n}$ belongs.

2) New point 2 for global fidelity.

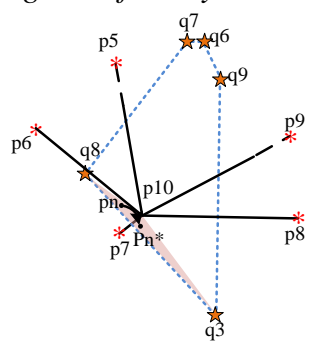

Figure 4. Addition of a new sampling point for the optimum.

For the global fidelity purpose, a region where existing sample points are depopulated must be effective. Under the terminology of Voronoi diagram, each Voronoi point is the farthest point from its surrounding sample points. A second new sample point is chosen based on the largest $\operatorname{dis}\left(p_{i}, q_{i}\right)$ and $q_{i}$ is the candidate new second point. Taking Figure 4 as an example, since $q_{6}$ is the farthest point to any surrounding $p$ points, it will be the second new sample point if needed.

\section{3) New point 3 between points 1 and 2}

Adding new samples at the above two types of points, the densities of samples around the tentative optima and around the most depopulated one become quite different. In order to connect the parts of both areas, a third new sample point is chosen in the middle of the interconnection of the above two samples or around 3/4 between the above two samples.

\section{CASE STUDIES}

The proposed approach is tested on the WSCC 9-bus system and the NPCC 140-bus power system. Accurate dynamic models with loads and the dynamic var sources are important for credibly simulating the phenomena of FIDVR and dynamic var supporting. The CLODBL load model and CSVGN5 SVC model (see [11] for details) are applied here. In the case studies, we assume all $c_{i}=1$, i.e. all buses have identical costs for SVCs, all $c_{p}=100$ in 9-bus system and $c_{p}=$ 1000 in NPCC system which will be larger than any single var upper limit, i.e. all buses have identical penalty for violating the voltage standard. In this case, the objective is actually to minimize the total amount of dynamic var supports to meet the voltage criteria. 


\section{A. 2-dimensional Searching Space for the 9-bus System}

The 9-bus system has 3 generators, 3 transformers, 3 loads, and 5 transmission lines, as shown in Figure 5. According to contingency analysis, a three-phase fault on bus 7, which is cleared by opening the line between buses 7 and 5 after 5 cycles, is identified as the most severe $\mathrm{N}-1$ contingency. Buses 6 and 8 with highest VSI are selected as two candidate buses to install SVCs. Assume the sizes of the two SVCs are larger than $1 \mathrm{Mvar}$ and the fidelity index is $\varepsilon=1 \mathrm{Mvar}$.

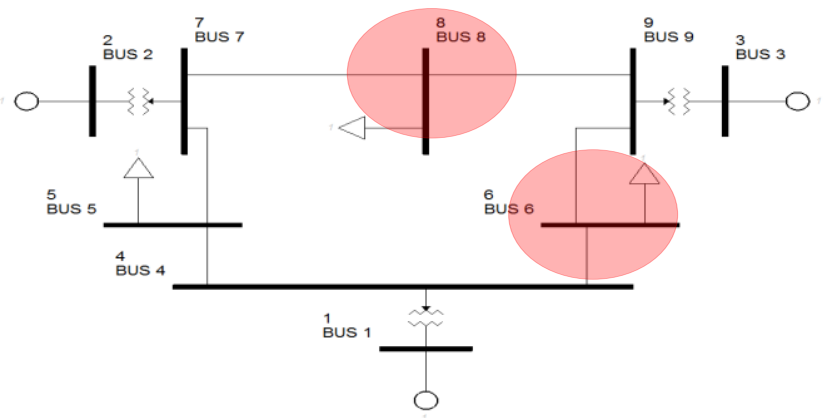

Figure 5. The 9-bus system with the most severe $\mathrm{N}-1$ contingency and two candidate buses for dynamic var supports.

Figure 6 shows how a contour map on the objective function is updated with new samples added and how the tentative optimal solution approaches to the actual global optimum. In Figure 6(a), a solution is gotten as $f=86$ Mvar at $\left(Q_{6}=85\right.$ Mvar, $Q_{8}=1$ Mvar $)$, under the initial approximation with ten sample points. The shapes of approximated functions are rather different from the true ones. In Figure 6(b), the solution is updated as $f=80 \mathrm{Mvar}$ at $\left(Q_{6}=71 \mathrm{Mvar}, Q_{8}=7 \mathrm{Mvar}\right)$, under the approximation with 13 sample points. The shapes of approximated functions are still different from the true ones, especially, in the bottom right zone. In Figure 6(c), the solution is moved to $f=$ $69 \mathrm{Mvar}$ at $\left(Q_{6}=68 \mathrm{Mvar}, Q_{8}=1 \mathrm{Mvar}\right)$. In Figures 6(d) and (e), the shapes of approximated function are further refined especially for the areas around the true optimum. The approximation improvement finally leads to a solution close enough to the true solution with $f=57 \mathrm{Mvar}$ at $\left(Q_{6}=\right.$ $56 \mathrm{Mvar}, Q_{8}=1 \mathrm{Mvar}$ ) in Figure 6(e) from $f=60 \mathrm{Mvar}$ at $\left(Q_{6}=58\right.$ Mvar, $\left.Q_{8}=2 M v a r\right)$ in Figure 6(d).

Figure 6 shows the history of the objective function and design variables over the number of function evaluation calls. This problem has a global optimum $f=57$ Mvar at $\left(Q_{6}=\right.$ 56 Mvar, $Q_{8}=1$ Mvar $)$ and the region of feasible solutions area is bounded by green color. Figure 7 shows the searching path of the tentative optimal as well as local optimal. The result is identical to the solution in [2] and [3].

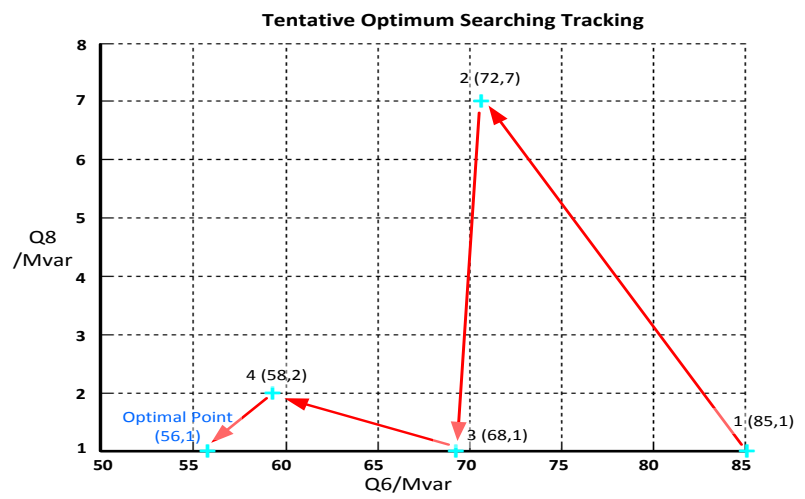

Figure 7. Searching path of the tentative optimum for the 9-bus system.

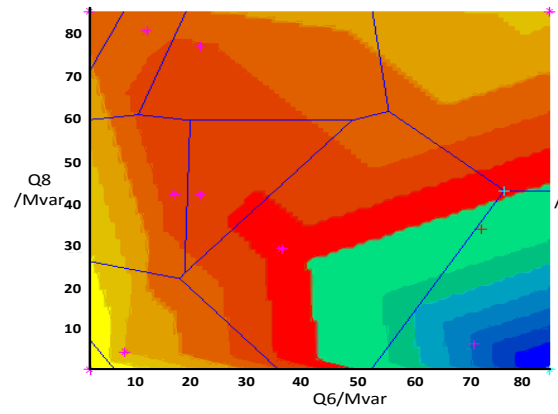

(a) Approximation with initial ten points

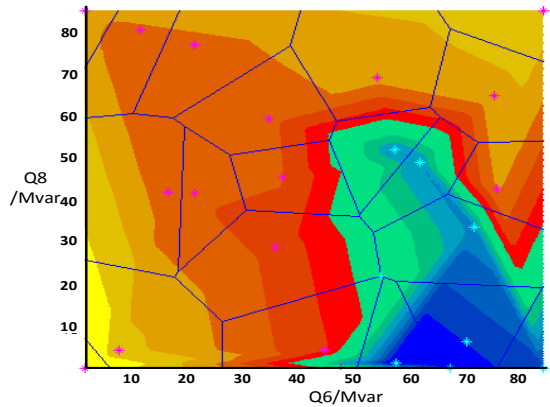

(d) Approximation with additional 12 points(22 in total)

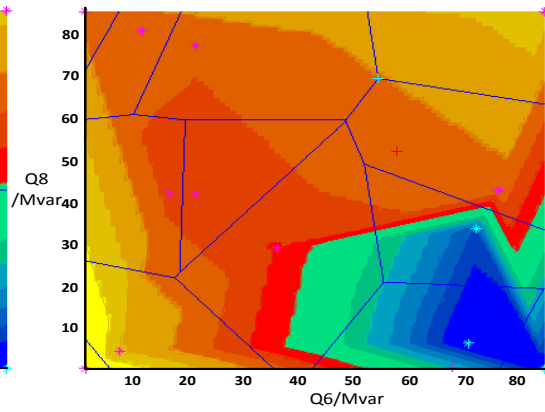

(b) Approximation with additional 3 points(13 in total)

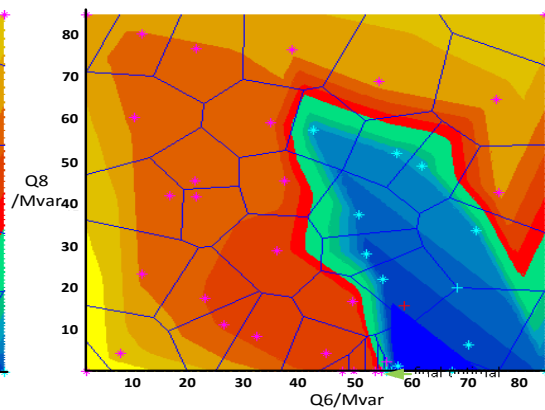

(e) Approximation with additional 30 points(40 in total)

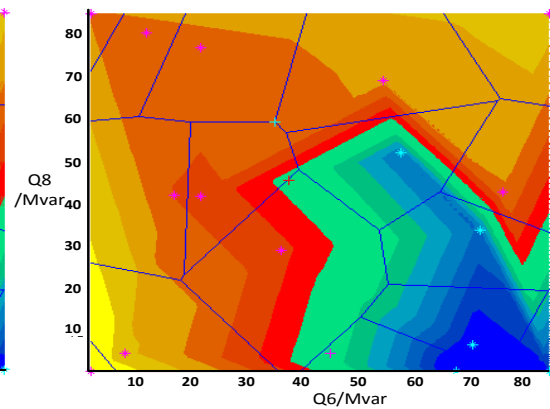

(c) Approximation with additional 6 points(16 in total)

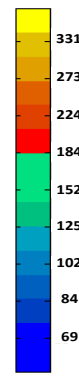

sample point violating low limit sample point within boundary

first addition point + tentative optimum second addition point most depopulated point

third addition point Linkage of first and second point $4 \quad$ final optimal

Figure 6. Contour map of approximated function during cumulative global optimization. 


\section{B. Three-dimensional Searching Space for the NPCC System}

As shown in Figure 8, the NPCC system is also used to test the proposed approach, which has 48 generators, 140 buses and represents the northeast region of the EI system. One of the most severe N-1 contingencies is identified as a three phase fault on bus 6 cleared by opening line 6-7 after 5 cycles. Buses 3, 6, and 31 are selected to be candidate buses to address the FIDVR issue in the system by voltage sensitivity index method. Assume that the sizes of SVCs are larger than $25 \mathrm{Mvar}$ and the fidelity index is $\varepsilon=5 \mathrm{Mvar}$.

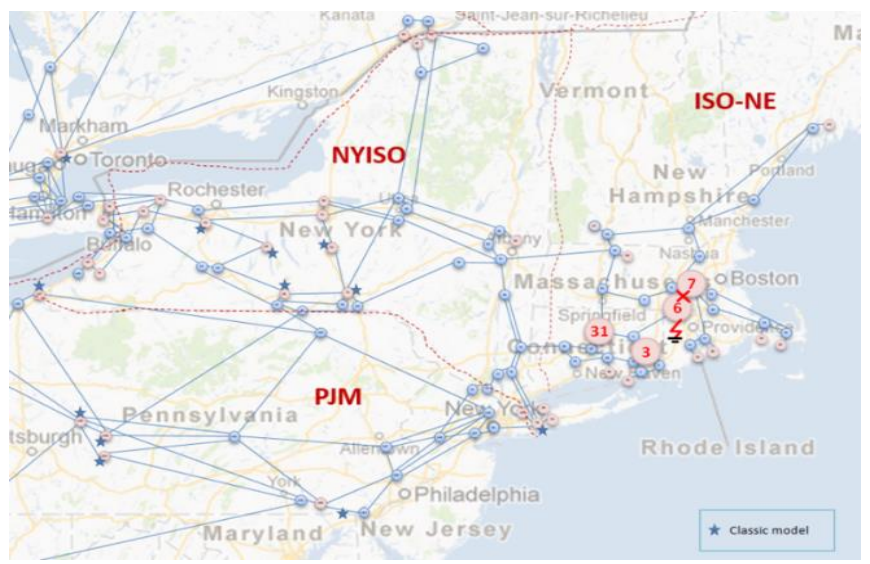

Figure 6. Part of the NPCC system and the most severe N-1 contingency and three candidate buses for dynamic var sources.

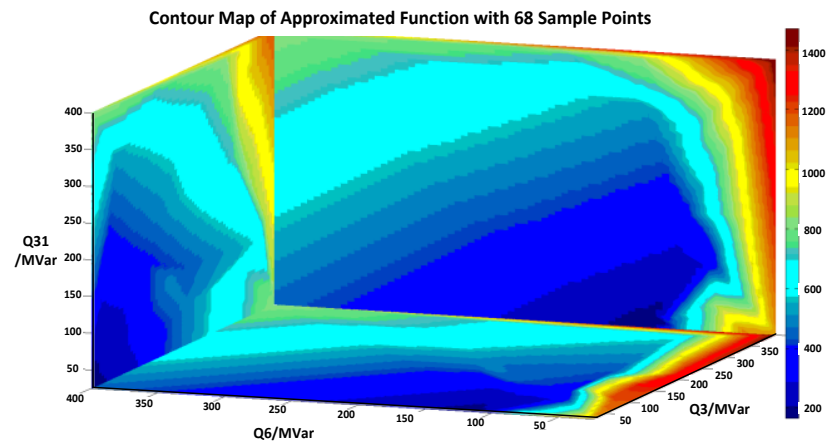

Figure 9. Contour map of approximated function with 68 sample points.

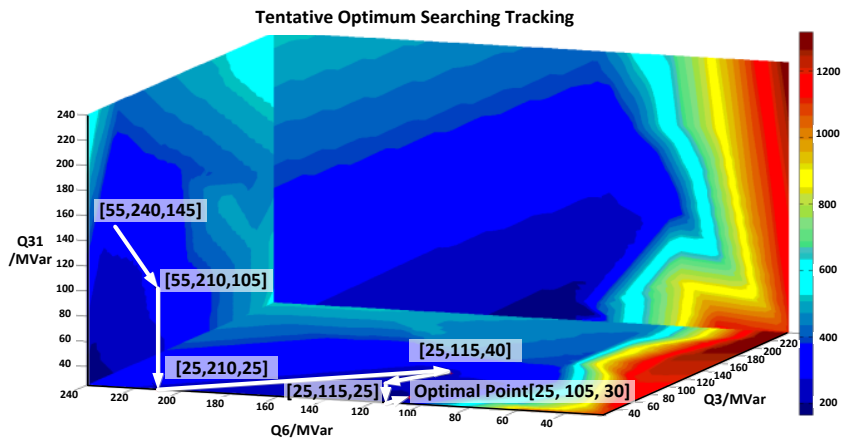

Figure 10. Searching path of tentative optimum for the NPCC system.
In order to view the plot clearly, the contour map is projected into three 2-D planes each about the sizes of two SVCs. Figure 9 shows a profile of the objective function in the searching space approximated based on 68 sample points, and the region of feasible solutions is bounded by green color within which a darker color indicates a smaller value of the objective function. The optimal solution is located on the right front bottom corner and it has $f=160 \mathrm{Mvar}, Q_{3}=25 \mathrm{Mvar}$, $Q_{6}=105 \mathrm{Mvar}$ and $Q_{31}=30 \mathrm{Mvar}$. Figure 10 shows the trajectory of iterations converging to the global optimum. This new approach gives the same optimum as the approach in [3].

\section{CONCLUSION}

This paper proposes a new approach to solving optimal sizes of dynamic var sources addressing FIDVR problems, which is a complex non-convex optimization problem. The new approach can identify the boundary of feasible solutions in a searching space and the globally optimal solution thanks to the Voronoi diagram and barycentric interpolation based objective approximation. Facing larger-scale problems, the computational burden of the new approach will increase with the growth of dimensions, i.e. the number of dynamic var sources. Future work will include testing the new approach on larger scale problems and incorporating this Voronoi diagram based approach with other optimization techniques.

\section{REFERENCES}

[1]. A. Tiwari and V. Ajjarapu, "Optimal allocation of dynamic var support using mixed integer dynamic optimization", IEEE Trans. Power Syst., vol. 26, no. 1, pp. 305-314, Feb. 2011.

[2]. M. Paramasivam, et al, , "Dynamic optimization based reactive power planning to mitigate slow voltage recovery and short term voltage instability", IEEE Trans. Power Syst., vol. 28, pp. 3865-3873, Nov. 2013.

[3]. W. Huang, K. Sun, J. Qi, Y. Xu, "A new approach to optimization of dynamic reactive power sources addressing FIDVR issues," IEEE PES General Meeting. National Harbor, MD, 2014.

[4]. S. Wildenhues, et al, "Optimal allocation and sizing of dynamic var sources using heuristic optimization", IEEE Trans. Power Syst., in press.

[5]. Y. Xu, et al, , "Dynamic optimization based reactive power planning to mitigate slow voltage recovery and short term voltage instability", IEEE Trans. Power Syst., vol. 29, no. 6, pp. 2813-2822, Nov. 2014.

[6]. M. G. O. Cheong, et al, "Computational Geometry: algorithms and applications (3rd ed.)," Springer-Verlag, New York, 2008.

[7]. N. Hirokawa, K. Fujita, and T. Iwase, "Voronoi diagram based blending of quadratic response surfaces for cumulative global optimization", 9th AIAA/ISSMO Symposium on Multi-Disciplinary Analysis and Optimization, Atlanta, Georgia, September 4-6, 2002.

[8]. J. Berrut and H. D. Mittelmann, "Matrices for the direct determination of the barycentric weights of rational interpolation," Journal of Computational and Applied Mathematics, 78 pp. 355-370, 1997.

[9]. NERC/WECC Planning Standards, WECC, Apr., 2003.

[10]. S. Cheng, T. K. Dey, and J. R. Shewchuk, Delaunay Mesh Generation, CRC press, Jan., 2013.

[11]. Siemens PTI Power Technologies Inc., PSS/E 33, Program Application Guide, vol. II, May 2011. 